\title{
Minimally invasive surgical approach for abdominal wall reconstruction. A new era in treatment of complex abdominal wall hernias
}

\author{
Cirugía mínimamente invasiva para la reconstrucción de \\ la pared abdominal. Una nueva era en el tratamiento de las hernias \\ complejas de la pared abdominal
}

Youri Mnouskin

Head of Advanced Laparoscopy Unit, Assuta Ashdod Public Hospital, Ben-Gurion University of the Negev; Ashdod, Israel.

This article aims to familiarize the general medical community with a history of the evolution and the wide range of new mini-invasive methods of surgical treatment for abdominal wall hernias. The indications, advantages, disadvantages, and possible prospects for the future will be discussed.

Since ancient times, the treatment of abdominal wall hernias has preoccupied the minds of surgeons. The wide range of surgical methods, proposed in due time, reflects the diversity in the traditions of various surgical schools and a lack of understanding of the physiology and anatomy of the abdominal wall. During the second half of the last century, abdominal wall surgery has made a significant step forward. This was facilitated by introducing synthetic mesh prosthesis and a fundamental understanding of abdominal wall anatomy. These advancements were made possible primarily through the experience gained during the repair of inguinal hernias.

In this regard, the most significant contribution to the development of modern abdominal wall surgery is associated with the names of three outstanding French surgeons who were ahead of their time. Only with the development of the laparoscopic technique came the general surgical community's realization of the true value of their heritage. The first of these, Henri Rene Fruchaud, published two books in 1956, in which he described the presence of a weak area in the groin and termed it as the myopectineal orifice. The second, Rene Stoppa, described the repair of

Keywords: minimally invasive surgical procedures; abdominal wall; hernia, abdominal; hernia abdominal; incisional hernia; reconstruction.

Palabras clave: procedimientos quirúrgicos mínimamente invasivos; pared abdominal; hernia incisional; reconstrucción.

Received date: 01/10/2021 - Acceptance date: 01/25/2021 - Published online: 03/30/2021

Corresponding author: Youri Mnouskin, MD; Head of Advanced Laparoscopy Unit, Assuta Ashdod Hospital Public Hospital, 7 HaRefu'ah St., 7747629 Ashdod, Israel. Telephone Number: 08-3004100. Email: drmanos90@yahoo.com

Cite as: Mnouskin Y. Minimally invasive surgical approach for abdominal wall reconstruction. A new era in treatment of complex abdominal wall hernias. Rev Colomb Cir. 2021;36:397-402. https://doi.org/10.30944/20117582.881

This is an open access article under a Creative Commons License - BY-NC-ND https://creativecommons.org/licenses/by-ncnd/4.0/deed.es 
bilateral, large scrotal, and recurrent inguinal hernias in 1965. Stoppa's technique included placing a prosthetic mesh at the pre-peritoneal plane over Fruchaud's myopectineal orifice, thus allowing reinforcement of the inguinal region's abdominal wall. Lastly, Jean Rives introduced the concept of Retro-Rectus Mesh Repair in 1966. Rives conducted his work independently of Stoppa and used a vertical incision in the lower abdomen to reach the groin regions, in contrast with Stoppa, who used a horizontal supra-pubic incision. Thus, he was able to isolate the retro-muscular space along the entire length, which made it possible to use this method to treat midline hernias. His method is still being used today as a gold standard repair of midline hernias and has been eponymously termed as the Rives Stoppa Repair (RSR).

With the advancement of laparoscopic equipment in the 1980s, hernia surgery became one of its first application areas. In 1993, two laparoscopic approaches were presented for the treatment of inguinal hernias. The first is the trans-abdominal approach, which involves opening the peritoneal sheath in the groin region via the peritoneal cavity to isolate the myopectineal orifice. This approach was termed Trans-Abdominal Pre-Peritoneal (TAPP). The second is the pre-peritoneal approach, which allows the same areas to be reached, but without entering the peritoneal cavity, thus termed Totally Extraperitoneal (TEP). These two prominent procedures share a common repair mechanism: incorporating a synthetic mesh according to the principles developed by Rene Stoppa. Each of these procedures has its advantages and disadvantages, as well as its specific indications for use. Furthermore, due to the substantial variability of the different abdominal wall and inguinal defects, for the successful treatment of any possible pathological variant, the hernia surgeon master both techniques.

The first attempts at laparoscopic treatment of anterior abdominal wall hernias were also made in the early nineties. The operation's principle was to release the herniated contents and cover the hernia orifice with a mesh, which was installed under the parietal leaf of the peritoneum like a puncture patch to fix a flat tire. The mesh's inner side was in direct contact with the abdominal contents, and the mesh was fixed to the abdominal wall using specially designed devices with a clip of self-fixing elements called tackers. The procedure received the acronym Intra-Peritoneal Onlay Mesh (IPOM), despite the incorrect designation of the mesh location (onlay implies the location above something, while the mesh is located below it). According to the modern nomenclature, such an arrangement of the mesh is called Underlay or Intra Peritoneal. Due to its relative simplicity and technical ease, the IPOM procedure quickly gained popularity among surgeons worldwide.

Nevertheless, unfavorable aspects of the IPOM were revealed early on. Insufficiently secured mesh tended to shrink and bulge in the defect area, which led to a rapid recurrence. Poor mesh fixation also resulted in the intestine entrapment between the mesh and the abdominal wall, resulting in bowel obstruction with subsequent necrosis. Furthermore, the mesh's intraperitoneal location allowed adhesion of abdominal contents to the mesh with subsequent intestinal erosions that led to entero-cutaneous fistula formation. This dreadful complication caused immense concern for surgeons and patients alike. These flaws in the IPOM technique were caused by multiple factors: the imperfection of the operating technique, lack of suitable meshes, and lack of proper fixation materials.

Numerous complications associated with meshes lead to increased demands for the development of new meshes and covering materials. Preventing mesh-related complications required proper implantation of the mesh onto the adjacent tissues on the outer side and the complete absence of adhesions between the mesh and the abdominal organs on the inner side. As a result, many meshes have been developed for these purposes by pharmaceutical companies, with a wide range of properties and costs, which may vary from hundreds to thousands of dollars per unit. Unfortunately, none of the companies managed to create a perfect IPOM mesh. Most of the offered products were discontinued. The development 
of new types of IPOM materials is currently suspended in most companies due to the emergence of more progressive methods in abdominal wall hernias' surgical treatment.

Nevertheless, a wealth of experience and understanding of the physiology of using mesh implants in the human body has been accumulated. The materials available today for IPOM repairs have overcome most of the flaws of the early meshes. They are relatively safe for use, with only a high price being their main disadvantage. A similar evolution has taken place with the production of mesh fixing devices. For a proper fixation, the principle of a "double crown" was proposed with the distribution of tackers both along the outer \& inner perimeter of the mesh covering the defect. This required a large number of fixing elements for one operation, each of which could potentially be a weak link leading to a negative outcome of the entire operation. Despite some of the disadvantages, the main issue with fixation devices is tissue injury, with severe pain syndrome both in the early and later postoperative period.

Concerning operating technique, to reduce mesh deformation, it was proposed to remove the pre-peritoneal fat layer in the area of mesh implantation, image speaking, to prepare the landing zone. These early experiences with laparoscopic abdominal wall repairs allowed a deeper understanding of the pathophysiology of hernia recurrence. This new understanding led to the obligatory suture closure of defects and the passing of additional fixating sutures, supporting the mesh through the abdominal wall's entire thickness- with the risk of developing pain at the suture site. The recurrence rate approached acceptable figures. The innovations introduced were combined into a single technique termed IPOM-plus. The old technique for greater separation is sometimes termed IPOM-bridge. The issues with the modern IPOM-plus remain the high cost, acute and chronic pain, and the almost inevitable adhesion process.

From the end of the eighties, surgeons are faced with another unresolved issue. The development of surgical technology and science has led to a widespread increase in the number of surgical interventions through the abdominal wall and, as a result, in a massive increase in the number of patients with large abdominal defects. Use of new surgical tactics, aimed for treating patients with major trauma or with critical conditions of the abdominal organs, required surgeons to manage patients without closing the abdominal wall for a long time and contributed to the emergence of a new type of abdominal wall pathology, known in the past only for rare examples of neglected untreated hernias. This condition, termed loss of domain, occurs when most of the abdominal cavity contents are located in the hernia sac; that is, the abdominal wall cannot keep the contents inside. Loss of domain leads to severe disability and, until recently, was considered practically inoperable. Midline hernias were further complicated by various conditions such as withdrawal of stomas through the abdominal wall following general or urological surgery.

None of the developed methods, both open and laparoscopic, allowed proper repair of defects measuring eight centimeters and larger diameter. Even the RSR did not allow for effective reconstruction of the abdominal wall with large and complex defects. This operation, like any other, has its anatomical limitations. The fascination with less invasive procedures, including laparoscopic ones, hindered the widespread dissemination of this progressive technique. Several methods have been proposed for resolving the issue in the old manner, with the implantation of inadequate supporting meshes and the lack of full approximation of the defect margins. Such techniques led to rapid recurrence in unacceptable percentages of cases. Attempts to approximate the hernial boundaries at any cost often led to an increase in intra-abdominal pressure after surgery with the development of concomitant conditions requiring emergency intervention. Overweight patients with large abdominal wall defects were considered inoperable. Surgeons at risk of choosing an operative way of treating such hernias presented their patients with unrealizable demands for weight loss and long-term physical activity limitation in the postoperative period. 
Plastic surgeons were involved in solving the problem of reconstructing the abdominal wall; thus, in 1990, Oscar Ramirez and colleagues proposed a technique termed component separation. This surgical technique is based on midline incision, subcutaneous lateral dissection, fasciotomy lateral to the rectus abdominis muscle, and dissection on the plane between external and internal oblique muscles with medial advancement of the block that includes the rectus muscle and its fascia. This release allows for medial advancement of the fascia and closure of up to $20 \mathrm{~cm}$ wide defects in the midline area. This technique's main disadvantage is the extended dissection of the cutaneous flaps, leading to ischemia and other wound complications. This technique has retained its relevance today, sometimes being the only possible access after several unsuccessful attempts to repair the abdominal wall by other methods. Muscle separation can be performed today in a minimally invasive approach to reduce wound complications. There remains the problem that the only possible plane for its installation turned out to be over anterior fascia, as an onlay repair according to the modern nomenclature. With this approach, the mesh was more prone to infection due to its proximity to the skin, and the lateral extension of the mesh is limited by the level of dissection of the cutaneous layer.

Nevertheless, Ramirez's discoveries paved the way for a breakthrough in abdominal wall surgery by separating the lateral element of the abdominal wall to better approximate the central block. Researches focused their attention on the RSR method, which involved dissection between the rectus muscles and the posterior fascia up to the semilunar line. Initially, it was suggested to continue the dissection more at the same level. The anatomical structure of fasciae seemed to allow for separation only between the internal oblique and transverse muscles. Positioning the mesh in this plane is termed retro oblique by modern nomenclature and has been abandoned. This type of procedure was termed Posterior Component Separation (PCS), while the Ramirez operation was given a new name-Anterior Component Separation (ACS). The main drawback of this pro- cedure soon emerged. For dissection between the internal oblique and the transverse muscles, it was necessary to cross the vascular and nerve branches feeding the rectus muscle, which led to their atrophy. The operation was accompanied by extensive bleeding due to the tight fusion between these two muscles, which did not allow enough approximation of the central block. The search seemed to have led to a dead end.

New research was required in the field of anatomy and physiology. The anatomy and function of individual elements of the abdominal wall and the abdominal wall's functioning as a whole organ were restudied. As a result, new surgical interventions were proposed, opening a new era in the reconstruction of the abdominal wall. The knowledge and experience accumulated by generations and greatly enhanced by the emergence of new technologies have led in the last decade to a real explosion in the field of abdominal wall surgery, like a new Cambrian. In 2012, Yuri Novitsky and his colleagues presented a new procedure: the Transversus Abdominis Release (TAR). Researchers based their technique on the finding that the TA muscle extends medially under the rectus muscle in the upper third of the abdomen. Division of the TA and dissection between this and the internal oblique muscle in a bloodless and devoid of nerves plane allows the closure of large defects. That made it possible to install large meshes to support the abdominal visceral compartment. This procedure turned out to be a logical extension of the RSR. The mesh's location away from the skin reduced the likelihood of infectious complications, and the lack of contact between the mesh and the viscera excluded the formation of adhesions, which made it possible to use inexpensive meshes. According to modern nomenclature, many surgeons consider this retro-muscular mesh location as the plane predetermined by God for the mesh placement.

Therefore, big surgery with the help of the foremother, reconstructive surgery, coped with the issue of large hernias. However, the use of such large-scale and complex operations seemed unjustified for treating medium and small uncomplicated hernias of the abdominal wall. How to adapt the 
preference for the minimally invasive procedures to the new techniques for abdominal wall reconstruction? By the end of the 2010s, surgeons had difficulty mastering the minimal access technique, significantly lagging behind traditional methods in both results and popularity among surgeons.

Then a real miracle happened. In 2012, Jorge Daes presented a modification of the totally extraperitoneal approach (TEP) for the treatment of large, complicated inguinal hernias with improved visibility and ergonomics. Up to this point, extraperitoneal access was considered difficult, with drawbacks such as limited working space, poor visibility, and bumping between instruments. TEP remained in the orbit of experts. Daes's modification consisted of access and dissection of the retro-muscular space much higher than the umbilical level and lateral to the linea alba, with the division of its natural boundaries (arcuate line) if necessary and flexible port set up. This modification gave an almost unlimited access to the myopectineal orifice area and the extraperitoneal space of the lower abdomen, a large working space, and the possibility of good triangulation of the camera and instruments. Daes made the maneuver suggested by Rives to use Stoppa's access for the midline lower abdominal hernias. Daes added a cursive letter $e$ to the standard access name, which means enhanced or extended. History has completed yet another round half a century-long and raised abdominal wall surgery to a new level.

In 2015, Igor Belyansky had progressed on the eTEP and used it to develop an endoscopic approach to retro-rectus Rives-Stoppa repair and PCS-TAR. Belyansky brilliantly coped with such repairs' possible technical difficulties, describing the crossover between retro-muscular cavities without damaging the peritoneum, the precise transaction of the transverse muscles, and a complex intra-corporeal suture. The new procedures were termed eTEP RS (Rives-Stoppa) and eTEP TAR.

The eTEP pioneers included five surgeons from four continents: Jorge Daes, Igor Belyansky, Yuri Novitsky, Victor Radu, and Ramana Balasubramaniam. They are also successful promoters of the new method.
eTEP allowed the repair of complex ventral hernias using the modern principles of AWR and allowed surgeons to understand better the boundaries of dissection of the extraperitoneal space included the myopectineal orifice. Recently, Edward Felix, with Daes, outlined the Critical View of the Myopectinial Orifice or the golden rules for safe minimally invasive access for the treatment of inguinal hernias, based on the best available evidence. These rules make the minimally invasive access to inguinal hernia repair easier to learn and available to surgeons early on. Today, successful work is underway to train residents in the second or third year of residency, which was previously considered impossible and inexpedient.

The fans of the trans-abdominal approach have not stayed idle and further developed the IPOM technique. This development involved dissection of the parietal leaf of the peritoneum and the establishment of the mesh in the pre-peritoneal plane, which made it possible to practically cope with fixing the mesh and avoiding its contact with the viscera. Previously, this was considered impracticable due to the fragility of the peritoneal layer and its tight fusion with the fascia. New types of operations received the traditional name TAPP, which today refers not only to inguinal hernias but also to hernias of the anterior abdominal wall and reflecting the trans-abdominal approach and the pre-peritoneal location of the mesh.

Robotics enthusiasts have played an important role in developing pre-peritoneal techniques, demonstrating with apparent ease the most complex types of dissections for the treatment of abdominal wall hernias at any localization, including para-stomal hernias. Today, robotic surgeons are successfully mastering the retro-muscular space using both trans-abdominal and eTEP access. Robotic technologies make it possible to perform dissections and sutures with filigree precision in areas that are sometimes limited by the traditional laparoscopic method. Many surgeons also recognize that working with a robot is more ergonomic, which is especially important when performing complex abdominal wall reconstructions, which sometimes require many hours of hard work. 
Robotic technology is not only a fashionable gimmick but also an independent field in minimally invasive surgery of abdominal wall pathology. To denote robotic operations, a cursive letter $r$ is added to each procedure's traditional name. Among the outstanding surgeons who popularize robotics to treat abdominal wall pathology can be distinguished Karl A. LeBlanc, Filip Muysoms, and Conrad Ballecer.

On an ongoing basis, new types of access and operations appear every year. The minimally invasive approach is successfully used to treat the rectus muscles' symptomatic diastasis with a subcutaneous approach and an onlay mesh; combined hybrid approaches can successfully cope with abdominal wall pathology variants. Unfortunately, there are also attempts to pass off variants of already known techniques as discoveries. However, mass surgical communities created based on social networks successfully differentiate each of the researchers' contributions in this exciting and progressive field of surgery. Social networks also help popularize new progressive methods. Success and failure are scrutinized almost online, making them irreplaceable in modern surgical science. Among the most popular and authoritative closed groups, one can single out the International Hernia Collaboration and The AWR Surgeons Community's Facebook pages.

Today, for the first time since the dawn of abdominal wall surgery, surgeons do not rely on new expensive technologies and materials from the medical industry to treat abdominal wall defects properly. On the contrary, systematic work is underway to simplify and reduce the cost of surgical treatment methods while improving the already existing excellent results. We use the most straightforward tools and materials, practically abandoned expensive fixation devices, and use available meshes and sutures. Perhaps in the near future, we will also abandon the use of implants entirely in many areas. Today, the development of plastics of the inner inguinal ring by minimally invasive access without meshes is successfully carried out. Our instruments today are a thorough knowledge of the abdominal wall's anatomy and physiology, donated by the outstanding surgeons of our time. Excellent optics and craftsmanship turn every operation into a fascinating anatomical tour, unlike one another.

We have learned to use strategies to prepare patients with complicated hernias as neoadjuvant therapy turns inoperable tumors into operable ones. The use of botulinum toxin and the method of progressive pneumo-peritoneum makes it possible to successfully operate on patients with loss of domain and produce minimally invasive access for hernias previously considered available only to open surgery. Today we have a sufficient arsenal of tools to solve almost any of the most challenging problems in the field of surgery of the abdominal wall. Systematic work is underway to study and solve chronic pain in the groin, both postoperative and associated with physical activity.

New technologies, despite promising results, require careful testing of time. For an adequate assessment, it is necessary to create a single database, conduct comprehensive researches with the involvement of a large number of international hernia centers. The need has matured to create such centers in each academic clinic for planned work on the study and implementation of new technologies in treating abdominal wall pathology. Despite the complexity of the tasks being solved, to this day, both in the public mind and among colleagues, hernia surgery remains a simple field of surgery, sometimes belittled by surgical professions such as cardiac surgery, transplant surgery, surgical oncology, and others. Nevertheless, for surgeons who are faced daily with treating complex pathology of the abdominal wall, the need for specialization, a thorough study of all aspects of the theory for the correct decision-making, and mastering the most complex operating technique is obvious. The wide variety of pathologies encountered requires an extensive theoretical and practical arsenal. We are looking forward to the future, where abdominal wall surgery will take its rightful place among equals.

The most important task at this stage is to popularize the most progressive, safe, and effective methods and apply them worldwide. We are committed to making them available to surgeons of all levels. This task becomes possible with the involvement of progressive teaching and training methods. 